\title{
Granulopoiesis and Neutrophil Homeostasis: A Metabolic, Daily, Balancing Act
}

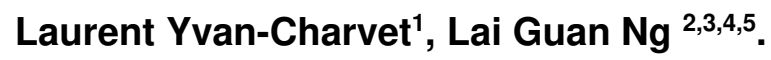

${ }^{1}$ Institut National de la Santé et de la Recherche Médicale (Inserm) U1065, Université Côte d'Azur, Centre Méditerranéen de Médecine Moléculaire (C3M), Atip-Avenir, Fédération Hospitalo-Universitaire (FHU) Oncoage, 06204 Nice, France.

2 Singapore Immunology Network (SIgN), A*STAR, Biopolis, Singapore

${ }^{3}$ State Key Laboratory of Experimental Hematology, Institute of Hematology \& Blood

Diseases Hospital, Chinese Academy of Medical Sciences, 288 Nanjing Road, Tianjin 300020, China

${ }^{4}$ School of Biological Sciences, Nanyang Technological University, 637551 Singapore ${ }^{5}$ Department of Microbiology \& Immunology, Immunology Programme, Life Science Institute, Yong Loo Lin School of Medicine, National University of Singapore, Singapore 117597, Singapore

Address correspondence to LYC: yvancharvet@unice.fr Address correspondence to NLG: Ng Lai Guan@immunol.a-star.edu.sg 


\begin{abstract}
:
Granulopoiesis is part of the hematopoietic hierarchic architecture where hematopoietic stem cells give rise to highly proliferative multipotent and lineage-committed granulocytic progenitor cells that differentiate into unipotent neutrophil progenitors. Given their short lifespan, neutrophils are rapidly cleared from circulation through specialized efferocytic macrophages. Together with an intrinsic clock, these processes contribute to circadian fluctuations, preserving self-tolerance and protection against invading pathogens. However, metabolic perturbation of granulopoiesis and neutrophil homeostasis can result in low-grade chronic inflammation, as observed with aging. During acute pathogenic infections, hematopoiesis can also be switched into emergency mode, which has been recently associated with significant neutrophil functional heterogeneity. This review focuses on a new reassessment of regulatory mechanisms governing neutrophil production, life-cycle and diversity in health and disease.
\end{abstract}




\section{A Day in the Life of a Neutrophil}

Neutrophils are the most abundant white blood cells in mammals, representing a first line of defense from daily environmental insults. However, due to their short half-life, more than $10^{11}$ mammalian neutrophils need to be replaced every day to maintain their roles as sentinels [16]. Blood production is a highly regulated process that originates from both spatial and timely organization of hematopoietic stem and progenitor cell (HSPC) self-renewal, expansion and differentiation in the bone marrow (BM) [1-6]. Using different cellular tracking approaches, it has been debated whether long-lived progenitors or short-term HSCs (ST-HSCs), rather than classically defined long-term HSCs (LT-HSCs), are the main drivers of steady-state hematopoiesis in the adult mammal [7-9]. Nevertheless, blood development starts with HSPCs that tailors the output of committed progenitor cells based on the specific hematopoietic need of the organism. Although neutrophils are the predominant leukocyte population in blood and are released from the BM at a steady rate under homeostatic conditions, questions remain regarding how HSPCs specify lineage fate towards granulopoiesis.

In this review, we primarily focus on the sequence of events involved in granulopoiesis downstream of the myelopoietic lineage, as well as the emerging factors that regulate the commitment and differentiation of this myelopoietic branch. Given accumulating evidence of neutrophil diurnal oscillations being coupled to circadian metabolism and immune surveillance to maintain tissue homeostasis, we discuss recent advances in our understanding of these new aspects of granulopoiesis regulation, examining granulopoiesis in the context of health and sterile inflammation, as in cases of chronic inflammatory diseases, inflammaging and cancer.

\section{Granulopoiesis: Neutrophil Production at Steady State.}

\section{Neutrophil Ontogeny}

Granulopoiesis involves a series of maturational steps from multipotent HSCs to lineagecommitted Common Myeloid Progenitor (CMP) cells, through differentiation, leading to the formation of granulocytes, including neutrophils. Seminal work identified and isolated CMP cells within a Lin-Sca-1 ${ }^{-} \mathrm{C}-\mathrm{Kit}^{+}$fraction (cellular phenotype) that could give rise to either megakaryocytes and erythroid cells, or macrophages and granulocytes -- depending on the expression of Fcy receptor-II/III (FcyR) and CD34 in mice (Key Figure, Figure 1) [10]. Even though these populations may still be heterogenous [11,12], they clearly represent two mutually exclusive intermediates in myelopoiesis; namely, the megakaryocyte/erythrocyte lineage-restricted progenitor $\left(\mathrm{FcyR}^{\circ}{ }^{\circ} \mathrm{CD} 34^{-} \mathrm{MEP}\right)$ or the granulocyte/macrophage lineagerestricted progenitor $\left(F c y R^{\text {hi }} \mathrm{CD} 34^{+} \mathrm{GMP}\right)$. Similarly, a myeloid-restricted progenitor population was identified in humans as being $\operatorname{Lin}^{-} \mathrm{CD} 34^{+} \mathrm{CD} 38^{+}$; with a IL3R $\alpha^{-} \mathrm{CD} 45 \mathrm{RA}{ }^{-}$ (double negative) fraction harboring an erythroid and megakaryocyte lineage potential, while the IL3R $\alpha^{+}$CD45RA ${ }^{+}$(double positive) fraction gave rise to cells of the granulocyte and macrophage lineages [13]-- with some heterogeneity within the two populations [14-16].

Stemming from a multi-omics integrative study [17], one report has elegantly characterized a specific multipotent progenitor 3 (MPP3) subset present within the Lin-Sca- $1^{+} \mathrm{c}-\mathrm{Kit}^{+}$(LSK) fraction of the mouse BM that is more efficient at producing GMPs with neutrophil-biased outputs than other MPPs (Key Figure, Figure 1) [18]. These mouse progenitors are produced by HSCs with specific kinetics depending on neutrophil demands [18]. Whether human Flk2 CD150-CD48+ MPP3 possess similar neutrophil-biased outputs remains to be clarified. At steady state, humans can produce up to 1 billion neutrophils per day per $\mathrm{kg}$ of body weight. Beyond its broad effect in producing active neutrophil proteases and transiently reorganizing the $\mathrm{BM}$ niche into a highly proteolytic environment, the granulocyte colony- 
stimulating factor (G-CSF) remains the main growth factor driving granulopoiesis across mammalian species [19-22]. Accordingly, three populations of unipotent neutrophil progenitors that respond to G-CSF in mice and humans, have been recently identified downstream of GMPs [23-25]. A hierarchical tree with these advanced committed neutrophil progenitors has also been recently proposed in mice [26]. Similar observations exist in humans, and a heterogenous $\mathrm{c}-\mathrm{Kit}^{+} \mathrm{CD} 66 \mathrm{~b}^{+} \mathrm{CD} 34^{+/}$cell population has been identified as an early neutrophil progenitor subset [24] -- presumably preceding the c-Kit-CD66 ${ }^{+}$CD34population [25] (Key Figure, Figure 1). One major characteristic of these proliferative neutrophil precursors across species is that they rapidly differentiate into mature $\mathrm{Ly}_{6 \mathrm{G}}{ }^{+} \mathrm{CXCR} 2^{+}$neutrophils, and this process involves several known transcriptions factors downstream of G-CSF, such as PU.1, C/EBP $\alpha$ or GFI1 [27]. Indeed, a recent study identified $\mathrm{C} / \mathrm{EBP} \varepsilon$ as a novel master transcriptional factor dictating neutrophil-exclusive commitment under homeostatic conditions in both mice and humans [25]. Collectively, these recent findings have helped demonstrate developmental pathways in granulopoiesis downstream of the myelopoietic lineage, and may provide a better understanding of how dysregulated granulopoiesis can impact immune homeostasis.

\section{C/EBPs Dominate Transcriptional Networks during Granulopoiesis}

The process of specification and differentiation of hematopoietic progenitors towards the myeloid lineage during resting state requires the coordinated activation of gene expression programs and myeloid transcription factors [28]. It is well established that transcription factors such as PU.1, RUNX1 and GFI1 play a fundamental role in granulopoiesis across species (see review [29]). Here, we focus specifically on the role of transcription factors belonging to the CCAAT enhancer- binding protein (C/EBP) family comprising six members $(\mathrm{C} / \mathrm{EBP} \alpha, \beta, \delta, \gamma, \varepsilon$ and $\zeta)$. Studies of CEBP family proteins have revealed that these are involved in numerous biological functions during homeostatic maintenance, as well as under pathological conditions [30]. Notably, each individual isoform has a specific role in granulopoiesis. Original analyses of genetically-modified mice lacking specific CEBP members have revealed a requirement for distinct C/EBP transcription factors during different developmental stages of granulopoiesis. The study of $c / e b p \alpha^{-/}$mice initially revealed neutrophil loss, and this deficiency was attributed to the failure of $\mathrm{C} / \mathrm{EBP} \alpha$ to regulate the G-CSF receptor (G-CSFR) promoter [31]. These findings are consistent with studies of $\mathrm{csf3}^{-/}$mice (i.e, G-CSF deficient mice) and $c s f 3 r^{-1-}$ mice (i.e, G-CSFR deficient mice) that are severely neutropenic [32,33]. Subsequent studies of genetically-modified animals and cell lines revealed that the lack of G-CSFR expression in C/EBP $\alpha$ deficiency could be restored by exogenous factors such as the mixture of granulocyte-macrophage colony-stimulating factor (GM-CSF), interleukin (IL)-3 and IL-6, to initiate C/EBP $\alpha$ independent granulopoiesis [34-36]. Given that GM-CSF, IL-3 and IL-6 serum concentrations are increased during inflammatory responses, it is reasonable to suggest that $\mathrm{C} / \mathrm{EBP} \alpha$ might be dispensable for granulopoiesis under inflammatory conditions such as acute infections. In contrast, a study has shown that in vitro cultures of C/EBP $\beta$-deficient hematopoietic cells have impaired responsiveness to GM-CSF [37]. This in turn suggested that $\mathrm{C} / \mathrm{EBP} \beta$ could contribute to $\mathrm{C} / \mathrm{EBP} \alpha$-independent granulopoiesis. Consistent with these findings, an elegant study illustrated the requirement of C/EBP $\beta$ in emergency granulopoiesis using both in vitro cultures and in vivo murine models of fungal infection or cytokine treatment [38]. Independent studies have also shown that $c / e b p \varepsilon^{-/}$mice are devoid of functionally mature neutrophils and have "atypical" granulocytes that lack secondary and tertiary granules [39,40]. Recent in-depth analysis using mass cytometry (CyTOF), cell-cyclebased analysis and transcriptomic profiling showed that neutrophil development is blocked at the pre-neutrophil stage in C/EBPE deficient mice, rendering these mice unable to either 
clear bacteria or mount immune complex-mediated inflammation [25]. While classical studies of knockout animals of different C/EBP members have provided insights into the role of $\mathrm{C} / \mathrm{EBP}$ proteins in granulopoiesis, this approach can be cell- and marker-biased, thereby lacking a global perspective of the transcriptional networks involved. Using single-cell RNA sequencing technology to map bone marrow myeloid progenitor subpopulations, several laboratories have established workflows to study transcriptional programs in myelopoiesis in an unbiased manner. These studies have provided a detailed view of HSC commitment into different leukocyte lineages with unprecedented resolution. Notably, one key concept arising from these transcriptomic and functional data at single-cell resolution is that mouse and human hematopoietic differentiation does not occur through a "rigid" step-wise manner, as previously thought, but rather, through a continuum pathway in which progenitor cells exist in transitional states with dynamic lineage potentials [41-43]. Moreover, recent single-cell studies combined with CRISP-seq (CRISPR-Cas9 sequencing) perturbation analyses showed that $\mathrm{C} / \mathrm{EBP} \alpha$ was required for progenitors to enter the myeloid lineage in mice, and furthermore, that subsequent differentiation and maturation towards the neutrophil lineage was dependent on the cooperation between C/EBPE and PU.1 [41,44]. In summary, we posit that these studies have formed an initial transcriptional framework for studying granulopoiesis under healthy or perturbed conditions.

\section{Neutrophil Circadian Oscillations}

Circadian rhythms are controlled by what are termed 'circadian clocks', present in every organ and every cell, and which synchronize an organism to anticipate daily environmental changes according to the day-night cycle. Leukocytes continuously circulate in the bloodstream under steady-state conditions and their circadian fluctuations have recently emerged as a critical mechanism aligning the body to the usual recurring cycles of the environment [45-49] (Figure 2). Although circulating immune cells display an opposite rhythm between mice and humans [50][51], peripheral neutrophils display daily oscillations in both species [50][51]. Most of the underlying mechanisms have been identified in mice (Box 1). One study revealed that circulating neutrophils exhibited robust circadian fluctuations in mice peaking a few hours after the initiation of light [52], paralleling earlier observations made for HSCs [53], or monocytes [54]. The mechanisms controlling the rhythmicity of mouse peripheral HSCs and monocytes involved oscillations in their BM retention by modulation of chemokines such as C-X-C motif chemokine 12 (CXCL12) within the BM microenvironment, or cell-autonomous control of chemokine receptors such as $\mathrm{C}-\mathrm{C}$ chemokine receptor type 2 (CCR2) in a clock-dependent manner [53,54][55]. An additional layer of regulation for neutrophil rhythmicity involves their unique pattern of aging in the blood and re-entry in the BM for clearance [52]. Specifically, murine neutrophil aging of in the circulation is characterized by reduced L-selectin (CD62L) expression and increased CXCR4 expression, mediating their homing back to the BM within 8-12 hours [52]. Although a recent report has questioned the estimated lifespan of neutrophils in circulation in mice [56-58], these findings are consistent with the expected short lifespan (8-12 hours) of neutrophils in both mice and humans $[59,60]$. Apart from their re-entry in the BM, aged murine neutrophils can also be cleared by specialized tissue-resident, as observed in the spleen $[61,62]$. Using a parabiosis approach of neutrophil reporter mice (conjoined with shared circulation), in addition to whole-mount imaging of cleared large intestine, a role of intestinal macrophages in clearing infiltrated neutrophils was also recently proposed [63]. Additionally, a slower rate of neutrophil aging was noted in germ-free mice, antibiotic-treated animals, or in mice with a specific myeloid deletion of the Toll-like receptor adaptor protein Myd88 (LysM-cre/Myd88 $8^{\mathrm{fl} / \mathrm{fl}}$ mice) indicated this process was driven by the activation of Toll-like receptor by the microbiota, relative to controls [64]. Recently, the functional importance of oscillatory expression of the endothelial intracellular cell adhesion molecule (ICAM-1) and vascular cell adhesion protein 1 (VCAM-1), was established for the rhythmicity of neutrophils and other 
leukocytes through their emigration from blood [65]. Specifically, the authors showed that neutrophils had a unique capacity to exhibit rhythmic homing to different tissues in mice; chronopharmacological blockade approaches revealed that L-selectin was required for neutrophil homing to the lymph node and spleen, while in the liver, a predominant role was attributed to VCAM-1 [65]. These findings extend existing knowledge of integrin and selectindependent specificity of neutrophil recruitment to various inflamed tissues (reviewed in [3]). Additionally, an intrinsic clock mechanism in neutrophils was identified by targeting the clock in mice with specific Bmal1 deficiency in myeloid cells, which displayed a lack of oscillations in the migration behavior of neutrophils to the spleen, along with altered expression of the nuclear receptor Nr1d1 (also known as Rev-Erbo) or the P-selectin glycoprotein ligand-1 (Psgl-1) relative to controls [65]. Bmal1 also controlled the expression of the chemokine CXCL2, as evidenced from chromatin immunoprecipitation (ChIP) of Bmal1 binding to E-boxcontaining promoter regions of $\mathrm{Cxcl}$. The relevance of this regulation was highlighted by the aged neutrophil phenotype and impaired diurnal recruitment to multiple tissues with myeloidspecific deficiency of Bmal1 and Cxcr2 (LysM-cre/Bmal/ $1^{f / f l}$ and $C x c r 2^{f / / f l}$ mice) relative to controls [66]. Furthermore, mice subjected to chronic sleep fragmentation developed myeloid expansion during the light period, reflecting an exacerbated production of the growth factor CSF1 relative to controls; this was prevented in mice with pre-neutrophil-specific deficiency in the hypocretin-receptor [67]. Human peripheral neutrophils also display daily oscillations paralleling time-dependent changes in superoxide production and phagocytic capacity [68]. These daily oscillations have been further correlated with synchronous fluctuations in plasma CXCL12 [68] but not cell surface expression of CXCR4 [65], suggesting that cell surface markers of aged neutrophils may differ between mice and humans. Although currently uninvestigated, the ratio of young vs. aged neutrophils might be expected to follow circadian oscillations in humans. Most neutrophils present in blood in the evening are likely to be young neutrophils mobilized from the BM, whereas in the morning, this subset may have already aged significantly and have returned to the BM or migrated to peripheral tissues; however, this remains to be investigated. It is reasonable to speculate that neutrophil circadian oscillations are most likely a part of the immune surveillance mechanisms set in place to maintain mammalian homeostatic tissue function (i.e, anti-microbial activity or elimination).

\section{Granulopoiesis during Inflammation and Disease}

\section{Neutrophil Heterogeneity}

The field of neutrophil plasticity is still in its infancy and most of the concept of neutrophil heterogeneity has emerged from the cancer field [69-71][72]. By contrast, The plasticity of macrophages has now been largely accepted and is reflected by their ability to sense, respond and rapidly adapt to their local microenvironment [73-77]. Nevertheless, Similar to macrophage studies, a helpful and simplified way for understanding neutrophil function is to arbitrarily divide these cells into two extreme phenotypes. These phenotypes consist of a pro-inflammatory $\mathrm{N} 1$ phenotype induced by IFN $\gamma$ (alone or in combination with LPS) and an alternative N2 phenotype induced after TGF $\beta$ stimulation, across species [78-80]. However, this classification certainly limits our ability to clearly define a boundary and categorize these cells during homeostasis, as neutrophils are likely to respond to steady-state environmental cues in a single or combinatorial fashion. Thus, it is important to note that such approaches cannot fully recapitulate the precise mechanisms and metabolic flows that govern neutrophil basic cellular functions and their heterogeneity in vivo (Box 1). Consistent with this notion, various subsets of neutrophils have been described in mice -- not only under steady-state $[81,82]$, but also under inflammatory conditions, such as CD49 $d^{+} V E G F R 1^{\text {hi }} C X C R 4^{\text {hi }}$ proangiogenic neutrophils $[83,84]$ or $\mathrm{CD} 11 \mathrm{~b}^{+} \mathrm{CD} 15^{\text {hi }} \mathrm{CD} 10^{-} \mathrm{CD} 16^{\text {lo }}$ 'hydrid' antigen-presenting neutrophils [85,86], among others [87-90]. Given the constant identification of infiltrated tissue neutrophil populations with novel functions, it is unclear whether these neutrophil 
subsets belong to separate granulopoiesis lineages or are simply a reflection of their propensity to sense environmental cues and adopt a wide array of phenotypes.

\section{Effects of Metabolism and Aging on Granulopoiesis}

Despite the original notion that HSCs are immortal and exempt from aging, it is now wellrecognized that the functional capacity and quantity of blood cells changes dramatically with age in both mice and humans (Box 1); this is accompanied by a decrease in the regenerative capacity of these cells, and a bias towards the myeloid lineage [5,91-93]. Over the last few years, several studies have identified an increased prevalence of somatic mutations that occurs with age in the blood and BM HSPCs of normal individuals, even in the absence of hematologic abnormalities. While this phenomenon does not typically give rise to noticeable symptoms, it does lead to increased risk for hematological cancers, infectious diseases and atherosclerotic cardiovascular diseases [94-99]. This common aging-related phenomenon has been named 'clonal hematopoiesis of indeterminate potential' (CHIP) [100]. While the origin of these mutations in the initiation of cancers remains unknown, [101], these might arise from a 'smoldering inflammation' known to impact hematopoietic stem cell fate in health and disease [102]; or, they might depend on telomere length dynamics [103]. Alternatively, numerous studies have shown that mitochondrial dysfunction, altered autophagy, replicative stress, and accrual of DNA damage all contribute to stem cell aging, at least in mice [104-107]. CHIP is linked to mutations in multiple genes encoding proteins such as methylcytosine dioxygenase 2 (TET2), DNMT3A and ASXL1 (reviewed elsewhere [108-111]). Dnmt3a and Tet2 deficient mice, or Dnmt3a ${ }^{-/}$and Tet2 ${ }^{-/}$bone marrow transplantation experiments were initially shown to promote HSPC expansion and a bias in lineage fate [112-115]. It is now well-recognized that epigenetic regulation is a driver of aging-associated HSC decline [116]. Together, these studies identify a potential mechanism for the origin of the bias towards the myeloid lineage with age, though it is yet unclear what underwrites granulopoiesis specificity, or how the latter may be related to altered neutrophil function.

The metabolic flexibility of myeloid progenitors is an integral component of many inflammatory diseases [117]. Studies initially showed that mouse models of hypercholesterolemia, defective cholesterol efflux pathways, or hyperglycemia, exhibited myelopoiesis, while bone marrow transplantation experiments revealed that deficiency in the main glucose transporter Glut1 limited glycolysis-dependent HSPC differentiation towards the myeloid lineage [118-121]. Cholesterol metabolism could also impact the osteoblastic and vascular endothelium niches to instruct HSPC fate [122,123]. Recently, intrinsic metabolic modulation of myelopoiesis progenitors (i.e, dual increase in glucose and cholesterol metabolism) was also revealed as an integral component of trained immunity [124]. Long-term epigenetic reprogramming of myelopoiesis progenitors could be recapitulated by feeding mice a Western type diet (WD), known to trigger myelopoiesis [125]. WD-induced myelopoiesis seemed to occur via direct and indirect mechanisms, such as cell extrinsic IL-1 $\beta$ signaling subsequent to peripheral macrophage toll-like receptor 4 (TLR4) activation, or cell-autonomous metabolic regulation of myeloid progenitors [126][127]. These findings raise questions regarding the interplay between 'hematometabolism' and epigenetics, as putative underlying mechanisms of accelerated myelopoiesis in many chronic inflammatory diseases, including those accompanying aging [128-130]. Of note, perturbation of the cellular Krebs cycle has been observed in many inflammatory diseases and Krebs cycle metabolites can act as central epigenetic modifiers -- with succinate and fumarate acting as antagonists of histone and DNA demethylases; alpha-ketoglutarate (aKG) as a cofactor of epigenomic dioxygenases such as TET2 and JmjC domain-containing demethylases [117]. In certain hematological malignancies, there is evidence that a shunt between bone marrow monocyte and neutrophil production can exist $[131,132]$; this suggests suggesting that dedicated signaling pathways for myeloid lineage specification may exist. Of relevance, during granulopoiesis and neutrophil differentiation, there are reports suggesting 
that mitochondrial respiration must be repressed; indeed, the phagocytic function of neutrophils and their relative rapid death compared to other immune cells have been linked to high glycolytic rate and low mitochondrial respiration in vitro [133,134]. However, a recent study using autophagy protein Atg7-deficient neutrophil precursors in vivo questioned this hypothesis because autophagy-dependent generation of free fatty acids (i.e, lipophagy) was shown to be critical for supporting fatty acid-dependent oxidative phosphorylation and normal neutrophil differentiation from neutrophil precursors [135]. Similarly, mice with neutrophilspecific deficiency in Atg5 exhibited increased neutrophil maturation relative to control littermates [136]; moreover, human HL-60 promyelocytic cell lines treated with the ATPsynthase inhibitor oliogomycin to reduce the electron transport chain flow, exhibited limited neutrophil differentiation in vitro relative to controls [137]. However, most of these studies have focused on neutrophil maturation without offering deep insights into the myelomonocytic branch; thus, dedicated metabolic pathways may emerge as central rheostat for granulopoiesis specification, especially during chronic inflammatory diseases.

\section{Aged and Metabolically-Perturbed Macrophages Can Impact Granulopoiesis}

As for HSC decline, there is emerging evidence of impaired innate and adaptive immune responses in the elderly -- a phenomenon referred to as 'inflammaging' [138-143]. Impaired macrophage effector function, along with hyperactivation and dysfunctional signaling, is a culprit of inflammaging that can be initiated by intrinsic and extrinsic effects [138-143]. This includes low-grade inflammation from acquisition of a senescence-associated secretory phenotype, or from chronic exposure to bacteria and bacterial products (e.g., endotoxin), e.g. secondary to increased intestinal permeability in mice (and perhaps in humans) $[143,144]$. One main effector function of macrophages is the clearance of apoptotic cells to support tissue integrity by destroying billions of dying cells everyday [145-148]. Of relevance, an in vivo study showed that homeostatic regulation of murine neutrophils relied on the clearance of dying neutrophils by specialized efferocytic macrophages located in peripheral tissues, such as the lung, bone marrow and spleen (following their entry via adhesion molecules e.g. integrins or selectins) [61]. Subsequently, when efferocytic cells ingested apoptotic neutrophils, IL-23 production was suppressed in these cells, leading to a feedback loop controlling G-CSF and granulopoiesis, and thus, regulating overall neutrophil numbers in blood [61,62]. Moreover, efferocytosis of secondary necrotic neutrophils was attenuated in peritoneal macrophages of aged mice relative to young mice, thus leading to prolonged peritoneal inflammation [149]. Of note, in certain chronic inflammatory diseases such as atherosclerosis, an inefficient clearance capacity of macrophages has also been observed when macrophages are metabolically perturbed, for example in mice with myeloidspecific deficiency of Glut1, cholesterol efflux transporters, Ucp2, or mitochondrial complex III [150-155]. Furthermore, we and others have recently observed that impaired clearance of apoptotic cells is linked to reduced lysosomal and mitochondrial functions $[156,157]$-- two processes that govern lipid handling by macrophages during efferocytosis [158]. Moreover, research into the resolution of inflammation has also identified new lipid signaling molecules secreted by macrophages and altered in aged mice[159], or with defective efferocytosis [160], currently termed 'metabokines' (such as lactate) [153] or 'pro-resolving lipid mediators' (e.g. resolvins). However, whether we can improve the efferocytic capacity of macrophages by resetting their metabolic efficiency, or by exploiting the pro-resolving activities of SPMs, remains to be determined; such putative manipulations are of interest, as if successful, they might enable mitigating the perturbed neutrophil homeostasis observed with age, and/or to overcome age-dependent effects on granulopoiesis.

\section{Emergency and Extramedullary Granulopoiesis during Inflammation}

While circulating neutrophil numbers are maintained at a constant number in the resting state, the hematopoietic system can rapidly switch to an 'emergency hematopoiesis' mode 
to increase neutrophil production to meet the demand for these cells during stress conditions (e.g. infection) (Figure 3). While the BM is the primary site for hematopoiesis at steady-state in adults (medullary), the spleen and liver can support extramedullary hematopoiesis $(E M H)$ across species during immune challenges, such as infection [128-130]. Specifically, the spleen has been shown to be a unique reservoir, able to supply additional myeloid cells continuously during sepsis or conditions of stress [161]. Emergency and extramedullary granulopoiesis involve a series of shared cascading events including: i) the sensing of pathogens through recognition of pathogen associated molecular patterns (PAMPs) derived from microorganisms by pattern recognition receptor (i.e. TLRs); ii) the release of granulopoietic cytokines and growth factors; and iii) the mobilization of neutrophils to effector sites as part of the 'fight-or-flight' reflex [162,163]. To support $E M H$, hematopoietic stem/progenitor cells (HSPCs) can circulate in the bloodstream for immunosurveillance [164] and can seed in extramedullary tissues under acute and chronic stress conditions (i.e, infection, myelofibrosis, myeloablation, pregnancy, anemia, myocardial infarction or cancer) [165]. A seminal study showed that HSPCs were localized in close proximity to Tcf21+ stromal cells within murine splenic red pulp and these cells could secrete niche factors such as CXCL12 and stem cell factor (SCF) to support splenic EMH [166]. Additionally, a novel transcription factor TIX1 has been identified that supports splenic EMH after LPS challenge in mice [167]. Indeed, conditions such as elevated G-CSF, GM-CSF, IL-3, FMS-like tyrosine kinase 3 (FLT3) ligand and IL-6 in mouse and human sera in response to infection, or induced IL-1 $\beta$ production upon adjuvant administration, suggests that these soluble factors can serve as 'relay signals' to potentiate increased neutrophil production after challenge, in both experimental mouse models and humans [163]. Other considerations regarding emergency granulopoiesis have been recently reviewed $[162,163]$.

G-CSF-dependent and independent emergency granulopoiesis can occur under conditions of acute inflammation. While it is well accepted that G-CSF is a prototypic neutrophil growth factor, there is a large body of literature showing that G-CSF is also a strong neutrophil mobilizer, especially under systemic inflammatory responses that trigger a profound increase in serum G-CSF. This is best exemplified by various experimental animal models and in humans, where a single dose of G-CSF injection is sufficient to increase neutrophil counts in peripheral blood, relative to controls [163]. The mobilization of neutrophils from BM into circulation has been studied best in experimental mouse models. These studies show that mobilization can occur through synergistic and/or distinct mechanisms, including i) CXCR2mediated mobilization [168]; ii) the release of thrombopoietin to potentiate neutrophil motility leading to neutrophil egress [169]; and iii) inhibition of CXCR4/CXCL12-mediated BM retention and lung margination of neutrophils [170-173]. However, under specific bacterial infections, G-CSF can also act as a negative regulator of neutrophil mobilization, as blocking G-CSF can elevate circulating neutrophil numbers through the inhibition of CXCR2-mediated mobilization relative to controls [174]. Paradoxically, results derived from experimental mouse models have also demonstrated that $c s f 3^{-/}$mice can still mount profound neutrophilia following Candida albicans infection, but yet, these animals remain more susceptible to candidiasis compared to wildtype (WT) infected mice[175]. Thus, this indicated that although G-CSF deficiency did not impact the development of neutrophilia after infection, neutrophil effector function could be impaired due to the lack of G-CSF production [175]. Of interest, GCSF has also been recently shown to induce fever through the modulation of prostaglandin E2 released from neutrophils, further supported by the observation that fever can be ameliorated in prostaglandin $\mathrm{E}$ synthase (mPGES-1/-)-deficient mice following G-CSF treatment relative to WT [176]. Collectively, these findings suggest that emergency granulopoiesis can occur in a G-CSF-independent manner in response to certain pathogens, 
and may require the adequate combination of different factors to efficient produce, mobilize, or lead to 'fight-or-flight' neutrophils. Overall, it is clear that G-CSF plays multifaceted biological roles in the generation/maintenance of neutrophils, which highlights the need to reassess the mechanisms of action of G-CSF in neutrophil development as well as its functions in these cells. In particular, it will be important to re-examine the outcomes of granulopoiesis under different types of infection in G-CSF deficient mice, relative to controls $[25,177]$.

\section{Granulopoiesis and Cancer}

In various mouse models of cancer, the spleen has been identified as a unique reservoir for generating tumor-associated neutrophils that can migrate towards tumors [178]. A study using a hepatoma mouse model showed that cancer-induced EMH could reprogram the function of hematopoietic stem/progenitor cells in the spleen to generate immunosuppressive neutrophils. This was based on the observation that splenectomy synergistically improved the efficacy of anti-(programmed death ligand 1) PDL-1 treatment in the tumor-bearing mice, relative to controls [179]. Although the BM niche and splenic stroma are quite different, neutrophil development in the spleen during $E M H$ still follows the same hierarchical developmental order as in the mouse BM [25]. Future studies should therefore determine what and how these factors lead to neutrophil differentiation when generated from EMH in the spleen versus the BM. G-CSF can also modulate granulopoiesis during malignancy (Box 1). For instance, in one study, metastatic breast cancer cell-derived G-CSF was also shown to reprogram myeloid precursors into retinoblastoma $\mathrm{Rb}^{\text {low }}$ immunosuppressive neutrophils in the mouse BM; furthermore, neutralization of G-CSF restored Rb expression in this model [180] (Figure 3). Apart from G-CSF, another key pro-inflammatory cytokine is soluble IL-1 $\beta$, which has been shown to induce neutrophil accumulation in the stomach tissue of a gastric cancer mouse model relative to controls [181]. Moreover, a recent study in mice provided evidence that distal lung cancer cells could modulate the BM niche, generating pro-tumoral Siglec- $\mathrm{F}^{+}$neutrophils, relative to controls [182]. Apart from such findings where tumor cells can modulate the subtype of neutrophils being generated, a recent report has also indicated that ovarian cancer cells can trigger early neutrophil recruitment to the omentum to establish the pre-metastatic niche [183]. Collectively, these studies demonstrate that cancer cells can produce local and systemic factors that not only modulate neutrophil production at intra- and extramedullary sites but also drive neutrophil recruitment and function within tumor sites. It will be interesting in the future to examine how neutrophil production and function are influenced by tumor types, and how neutrophils that are recruited to establish a premetastatic niche may impact tumor metastatic processes. We posit that with such an increased understanding, it may be possible to contribute to devising putative neutrophilbased therapeutic interventions to treat certain cancer patients.

\section{Concluding Remarks}

As discussed in this review, granulopoiesis represents one of the most important biological processes for generating neutrophils as a first line of defense from daily environmental insults. Here, we have provided an overview of the immune contexture of granulopoiesis (Key Figure, Figure 1), which is determined by many factors including immune status, circadian rhythms and metabolic processes. Numerous recent studies have helped to increase our understanding of these vital regulatory mechanisms. Based on these findings, a complex picture is emerging, comprising a continuum-based hierarchical program of 
granulopoiesis and of neutrophil functional heterogeneity; the ultimate appropriate outcome is a transcriptionally- and metabolically-controlled protective granulopoietic response.

However, there are still many unanswered questions about these processes (see outstanding questions). This is in part due to our incomplete understanding of neutrophil ontogeny, differentiation and phenotypic/functional heterogeneity. With the recent identification of neutrophil precursors and advanced knowledge of neutrophil heterogeneity, coupled to improved animal models, genomics, bioinformatics and imaging technologies, new lines of research in the field of neutrophil biology may emerge. These approaches may help to dissect neutrophil roles in immunodeficiencies and immuno-dysregulated conditions, contributing to the development of potential therapeutic interventions for neutrophil-related diseases. Accordingly, future research will need to comprehensively tease apart the relative 'hematometabolic' and circadian perturbations affecting granulopoiesis in acute and chronic inflammatory diseases, inflammaging, or cancer. Indeed, it might be possible to develop novel chronopharmacological approaches to regulating granulopoiesis. A special emphasis should be placed on determining the most appropriate experimental biomarkers to be used in measuring -if at all possible -- inefficient neutrophil immunosurveillance. Why is this important? Most of our knowledge in the clinic is based on neutrophil counts, yet we are now truly appreciating the complexity of granulopoiesis in health and disease. Indeed, many different inflammatory conditions or pathologies might converge in their molecular underpinnings, perhaps sharing common transcriptional and metabolic pathways that lead to inefficienct neutrophil responses. These in turn, might or might not be related to perturbations in emergency or extramedullary granulopoiesis. Indeed, this concept has just been recently illustrated by the demonstration of metabolic alterations and over-expression of fatty acid transporter protein 2 in neutrophils from certain cancers [184]. Altogether, we envision new discoveries emerging from studies such as these, where the neutrophil no longer represents a 'forgotten cell' within the myeloid cell family. 


\section{Box 1. Clinician's corner}

- The maintenance of constant circulating neutrophil numbers requires an exquisite balance between granulopoiesis and neutrophil mobilization into the circulation as well as efficient efferocytosis. This represents key intervention points for potentially treating perturbations in neutrophil homeostasis such as neutropenia or neutrophilia.

- Hematopoietic stem cell transplantation patients often remain neutropenic for weeks. Recently-identified proliferative neutrophil precursors may serve as a source for generating neutrophils, which might help bridge treatments by aiming to avoid prolonged periods of neutropenia.

- The immune response weakens during aging and chronic Inflammatory diseases, and can be accompanied by a bias towards myeloid lineage differentiation; there is a wide array of phenotypes that neutrophils can adopt under these circumstances. Strategies aimed at limiting myelopoiesis or reprograming efficient neutrophil functions might constitute a new way to enable fighting against certain diseases.

- Neutrophils are the most abundant leukocytes in the blood. An improved knowledge of neutrophil phenotypic heterogeneity might lead to the development of blood-based candidate biomarkers for certain inflammatory diseases, including cancer.

- Protein factors within tumor microenvironments have been shown to modulate granulocyte properties. Conceivably, the modulation of systemic factor(s) in cancer patients might allow the reprogramming of pro-tumoral neutrophils into anti-tumoral neutrophils.

\section{Glossary}

Accelerated myelopoiesis: event causing increased (above normal) production of bone marrow myeloid leukocytes (i.e, white blood cells), including neutrophils.

Autophagy: cellular detoxification process that disassembles unnecessary or dysfunctional cellular components for recycling.

Common Myeloid Progenitor (CMP): hematopoietic progenitor that gives rise to myeloid leukocytes.

Clonal hematopoiesis of indeterminate potential (CHIP): common age-related phenomenon where hematopoietic stem and progenitor cells acquire a non-tumor mutation contributing to the clonal formation of a genetically-distinct subpopulation of leukocytes.

Efferocytic macrophages: Macrophages are professional phagocytic cells that daily remove billions of apoptotic and necrotic (dying/dead) cells in our body.

Emergency hematopoiesis: Rapid increased production of hematopoietic cells (i.e, white blood cells or leukocytes) due to, for example, infection.

Extramedullary hematopoiesis $(\mathrm{EMH})$ : Production of hematopoietic cells (i.e, white blood cells or leukocytes) occurring outside of the bone marrow under physiopathologic conditions. 
Long-term HSCs (LT-HSCs): Quiescent hematopoietic stem cells, capable of self-renewal; can give rise to all hematopoietic lineages at least four months after transplantation.

short-term HSCs (ST-HSCs): Proliferative hematopoietic stem cells that can differentiate into all types of blood cells within four weeks.

Metabokines: Signaling molecules derived from metabolic processes; bearing paracrine and autocrine effects.

Multipotent progenitors (MPP): Hematopoietic progenitor cells that maintain a limited capacity for self-renewal compared with LT-HSC and ST-HSC but are capable of differentiating into all cell types of the hematopoietic system.

Pathogen associated molecular patterns (PAMPs): highly conserved structural components of microbes; recognized by pattern-recognition receptors.

Pattern recognition receptor: detect microbial pathogens via the recognition of characteristic microbial molecular motifs.

Pro-resolving lipid mediators: large, growing class of cell signaling molecules formed in cells via the metabolism of polyunsaturated fatty acids (PUFA).

Smoldering inflammation: Chronic exposure to irritant particles or inherited gene mutations causing exacerbated inflammatory macrophage responses

Replicative stress: event during DNA replication when the genome is exposed to various types of stress, and can result in replication stress (i.e, activation of checkpoint signaling).

Senescence-associated secretory phenotype: Cellular senescence is a phenomenon by which normal cells cease to divide and acquire an inflammatory secretome characterized by inflammatory cytokines, growth factors and proteases.

Tcf21 ${ }^{+}$stromal cells: primarily found around sinusoids in the red pulp of the bone marrow.

Trained immunity: the ability of an innate immune cell (in the absence of antigen receptor recognition) to develop an increased and protective immunological (trained) response against second inflammatory challenge such as infection.

Toll-like receptor: recognizes conserved microbial pathogens via recognition of characteristic microbial lipopolysaccharide (LPS) motifs

Acknowledgements. We are grateful to members of our laboratories for continued discussions, reflected in this review. We also apologize to the many colleagues whose contributions could not be discussed in this manuscript due to the word limitation.

This work was supported by grants from the Inserm Atip-Avenir program, the Agence Nationale de la Recherche (ANR-14-CE12-0017-01) and the European Research Council (ERC) consolidator program (ERC2016COG724838) to L.Y.C. This article is supported by Singapore Immunology Network ( ${ }^{*}$ STAR) core funding to L.G.N.

\section{Conflicts of interest. None}




\section{Figure Legends}

Key Figure, Fig. 1 Hierarchy of Granulopoietic Development in Mice and Humans.

Schematic illustration of how neutrophils are generated from committed progenitors that originate from hematopoietic stem and progenitor cells (HSPCs) in the bone marrow. LTHSC, long-term hematopoietic stem cells, reconstituting, self-renewing; ST-HSC, short-term hematopoietic stem cell; MPP, multipotent progenitors with limited self-renewal leading to transient but multilineage reconstitution; CMP, common myeloid progenitor; GMP, granulocyte/macrophage progenitor. Surface markers used for isolation are indicated in the white boxes for mouse, and yellow boxes/green font for human.

\section{Fig. 2 Circadian Rhythmic Production and Clearance of Neutrophils in Mice.}

Peripheral neutrophils exhibit robust circadian fluctuations peaking a few hours after the initiation of light. Three mechanisms have been implicated in this rhythmicity: i) Approximately $10^{11}$ neutrophils per day are produced in the bone marrow and their release is controlled by circadian oscillations of chemokines such as C-X-C motif chemokine 12 (Cxcl12), under G-CSF regulation and the Bmal1-dependent intrinsic clock (also dependent on other factors, as indicated); ii) Short-lived (8-12 h) neutrophils also dictate their fluctuations and (as opposed to young ( $\left.\mathrm{CD}^{2} \mathrm{~L}^{+} \mathrm{CXCR} 4^{-}\right)$), when neutrophils age $\left(\mathrm{CD}^{2} 2 \mathrm{~L}^{-} \mathrm{CXCR} 4^{+}\right)$, they are cleared by professional efferocytic cells (i.e, mainly macrophages) in the spleen, and potentially, in the intestine. lii) When efferocytic cells ingest apoptotic neutrophils, IL-23 production is suppressed, leading to a feedback loop involving Th17 helper T cells (pink cells), controlling G-CSF and the subsequent production and release of neutrophils into the bloodstream. Black cell: phagocytosed neutrophil. Blue cell: macrophage. The process of neutrophil aging or their removal from the circulation might be driven by the activation of Tolllike receptor signaling by the microbiota [64]. Additionally, oscillatory expression of endothelial ICAM-1 (and other cell adhesion molecules) accompanies the circadian rhythmicity (white boxes with waves) of neutrophil extravasation or homing (i.e, L-selectin is required for neutrophil homing to the spleen, while in the liver, a predominant role has been attributed to VCAM-1) [65]. HSPCs: hematopoietic stem and progenitor cells. White boxes with single lines: cleared neutrophils.

\section{Fig. 3 Beyond Homeostatic Granulopoiesis in Mice and Humans.}

While circulating neutrophil numbers are maintained at a constant level during resting state, the hematopoietic system can rapidly switch into an 'emergency' mode to increase neutrophil production and meet the demand for these cells during acute pathogenic infections. This 'fight-or-flight' reflex process involves a series of cascading events beyond the prototypic GCSF growth factor. Combined serum elevation of GM-CSF, IL-3 or FLT3 in response to infection is required to dictate bone marrow and extra-medullary granulopoiesis. In the case of tumorigenesis, G-CSF and potentially other inflammatory cytokines derived from cancer cells can also reprogram myeloid precursors in the bone marrow and spleen into neutrophils

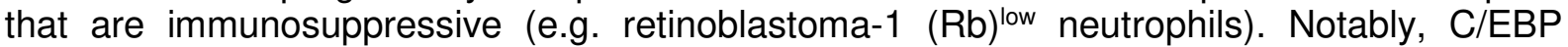
master transcription factor family members play a central role in dictating neutrophilexclusive lineage commitment under both homeostatic and emergency conditions [185]. 


\section{REFERENCES}

1 Mirantes, C. et al. (2014) Pro-inflammatory cytokines: emerging players regulating HSC function in normal and diseased hematopoiesis. Exp. Cell Res. 329, 248-254

2 Ema, H. et al. (2014) Heterogeneity and hierarchy of hematopoietic stem cells. Exp. Hematol. 42, 74-82.e2

3 Deniset, J.F. and Kubes, P. (2016) Recent advances in understanding neutrophils. F1000Res 5, 2912

$4 \quad$ Ito, K. and Frenette, P.S. (2016) HSC Contribution in Making Steady-State Blood. Immunity 45, 464-466

5 Weiskopf, K. et al. (2016) Myeloid Cell Origins, Differentiation, and Clinical Implications. Microbiol Spectr 4,

6 Nicolás-Ávila, J.Á. et al. (2017) Neutrophils in Homeostasis, Immunity, and Cancer. Immunity 46, 15-28

$7 \quad$ Sun, J. et al. (2014) Clonal dynamics of native haematopoiesis. Nature 514, 322-327

8 Busch, K. et al. (2015) Fundamental properties of unperturbed haematopoiesis from stem cells in vivo. Nature 518, 542-546

9 Sawai, C.M. et al. (2016) Hematopoietic Stem Cells Are the Major Source of Multilineage Hematopoiesis in Adult Animals. Immunity 45, 597-609

10 Akashi, K. et al. (2000) A clonogenic common myeloid progenitor that gives rise to all myeloid lineages. Nature 404, 193-197

11 Warren, L. et al. (2006) Transcription factor profiling in individual hematopoietic progenitors by digital RT-PCR. Proc. Natl. Acad. Sci. U.S.A. 103, 17807-17812

12 Paul, F. et al. (2015) Transcriptional Heterogeneity and Lineage Commitment in Myeloid Progenitors. Cell 163, 1663-1677

13 Manz, M.G. et al. (2002) Prospective isolation of human clonogenic common myeloid progenitors. Proc. Natl. Acad. Sci. U.S.A. 99, 11872-11877

14 Edvardsson, L. et al. (2006) Isolation and characterization of human myeloid progenitor populations--TpoR as discriminator between common myeloid and megakaryocyte/erythroid progenitors. Exp. Hematol. 34, 599-609

15 Mori, Y. et al. (2009) Identification of the human eosinophil lineage-committed progenitor: revision of phenotypic definition of the human common myeloid progenitor. $J$. Exp. Med. 206, 183-193

16 Mori, Y. et al. (2015) Prospective isolation of human erythroid lineage-committed progenitors. Proc. Natl. Acad. Sci. U.S.A. 112, 9638-9643

17 Cabezas-Wallscheid, N. et al. (2014) Identification of regulatory networks in HSCs and their immediate progeny via integrated proteome, transcriptome, and DNA methylome analysis. Cell Stem Cell 15, 507-522

18 Pietras, E.M. et al. (2015) Functionally Distinct Subsets of Lineage-Biased Multipotent Progenitors Control Blood Production in Normal and Regenerative Conditions. Cell Stem Cell 17, 35-46

19 Metcalf, D. (2008) Hematopoietic cytokines. Blood 111, 485-491

20 Greenbaum, A.M. and Link, D.C. (2011) Mechanisms of G-CSF-mediated hematopoietic stem and progenitor mobilization. Leukemia 25, 211-217

21 Lapid, K. et al. (2008) Egress and Mobilization of Hematopoietic Stem and Progenitor Cells: A Dynamic Multi-facet Process. In StemBook Harvard Stem Cell Institute

22 Bendall, L.J. and Bradstock, K.F. (2014) G-CSF: From granulopoietic stimulant to bone marrow stem cell mobilizing agent. Cytokine Growth Factor Rev. 25, 355-367

23 Kim, M.-H. et al. (2017) A late-lineage murine neutrophil precursor population 
exhibits dynamic changes during demand-adapted granulopoiesis. Sci Rep 7, 39804

24 Zhu, Y.P. et al. (2018) Identification of an Early Unipotent Neutrophil Progenitor with Pro-tumoral Activity in Mouse and Human Bone Marrow. Cell Rep 24, 2329-2341.e8

25 Evrard, M. et al. (2018) Developmental Analysis of Bone Marrow Neutrophils Reveals Populations Specialized in Expansion, Trafficking, and Effector Functions. Immunity 48, 364-379.e8

26 Ley, K. et al. (2018) Neutrophils: New insights and open questions. Sci Immunol 3,

27 Ng, L.G. et al. (2019) Heterogeneity of neutrophils. Nature Reviews Immunology

DOI: $10.1038 / \mathrm{s} 41577-019-0141-8$

28 Zhang, D.X. and Glass, C.K. (2013) Towards an understanding of cell-specific functions of signal-dependent transcription factors. J. Mol. Endocrinol. 51, T37-50

29 Cowland, J.B. and Borregaard, N. (2016) Granulopoiesis and granules of human neutrophils. Immunol. Rev. 273, 11-28

30 Ramji, D.P. and Foka, P. (2002) CCAAT/enhancer-binding proteins: structure, function and regulation. Biochem. J. 365, 561-575

31 Zhang, D.E. et al. (1997) Absence of granulocyte colony-stimulating factor signaling and neutrophil development in CCAAT enhancer binding protein alpha-deficient mice. Proc. Natl. Acad. Sci. U.S.A. 94, 569-574

32 Lieschke, G.J. et al. (1994) Mice lacking granulocyte colony-stimulating factor have chronic neutropenia, granulocyte and macrophage progenitor cell deficiency, and impaired neutrophil mobilization. Blood 84, 1737-1746

33 Liu, F. et al. (1996) Impaired production and increased apoptosis of neutrophils in granulocyte colony-stimulating factor receptor-deficient mice. Immunity 5, 491-501

34 Zhang, P. et al. (1998) Upregulation of interleukin 6 and granulocyte colonystimulating factor receptors by transcription factor CCAAT enhancer binding protein alpha (C/EBP alpha) is critical for granulopoiesis. J. Exp. Med. 188, 1173-1184

35 Collins, S.J. et al. (2001) Multipotent hematopoietic cell lines derived from

C/EBPalpha(-/-) knockout mice display granulocyte macrophage-colony-stimulating factor, granulocyte- colony-stimulating factor, and retinoic acid-induced granulocytic differentiation. Blood 98, 2382-2388

36 Zhang, P. et al. (2002) Induction of granulocytic differentiation by 2 pathways. Blood 99, 4406-4412

37 Akagi, T. et al. (2008) Impaired response to GM-CSF and G-CSF, and enhanced apoptosis in C/EBPbeta-deficient hematopoietic cells. Blood 111, 2999-3004

38 Hirai, H. et al. (2006) C/EBPbeta is required for "emergency" granulopoiesis. Nat. Immunol. 7, 732-739

39 Yamanaka, R. et al. (1997) Impaired granulopoiesis, myelodysplasia, and early lethality in CCAAT/enhancer binding protein epsilon-deficient mice. Proc. Natl. Acad. Sci. U.S.A. 94, 13187-13192

40 Lekstrom-Himes, J. and Xanthopoulos, K.G. (1999) CCAAT/enhancer binding protein epsilon is critical for effective neutrophil-mediated response to inflammatory challenge.

Blood 93, 3096-3105

41 Paul, F. et al. (2016) Transcriptional Heterogeneity and Lineage Commitment in Myeloid Progenitors. Cell 164, 325

42 Olsson, A. et al. (2016) Single-cell analysis of mixed-lineage states leading to a binary cell fate choice. Nature 537, 698-702

43 Velten, L. et al. (2017) Human haematopoietic stem cell lineage commitment is a continuous process. Nat. Cell Biol. 19, 271-281

44 Giladi, A. et al. (2018) Single-cell characterization of haematopoietic progenitors and their trajectories in homeostasis and perturbed haematopoiesis. Nat. Cell Biol. 20, 836-846 
45 Arjona, A. et al. (2012) Immunity's fourth dimension: approaching the circadianimmune connection. Trends Immunol. 33, 607-612

46 Scheiermann, C. et al. (2013) Circadian control of the immune system. Nat. Rev. Immunol. 13, 190-198

47 Curtis, A.M. et al. (2014) Circadian clock proteins and immunity. Immunity 40, 178186

48 Man, K. et al. (2016) Immunity around the clock. Science 354, 999-1003

49 Scheiermann, C. et al. (2018) Clocking in to immunity. Nat. Rev. Immunol. 18, 423-

437

50 Zhao, Y. et al. (2017) Uncovering the mystery of opposite circadian rhythms between mouse and human leukocytes in humanized mice. Blood 130, 1995-2005

51 Pick, R, He,W, Chen,CS and Scheiermann, C. Trends Immunol (2019) 40, 6: https://doi.org/10.1016/j.it.2019.03.010

52 Casanova-Acebes, M. et al. (2013) Rhythmic modulation of the hematopoietic niche through neutrophil clearance. Cell 153, 1025-1035

53 Méndez-Ferrer, S. et al. (2008) Haematopoietic stem cell release is regulated by circadian oscillations. Nature 452, 442-447

54 Nguyen, K.D. et al. (2013) Circadian gene Bmal1 regulates diurnal oscillations of Ly6C(hi) inflammatory monocytes. Science 341, 1483-1488

55 Chong, S.Z. et al. (2016) CXCR4 identifies transitional bone marrow premonocytes that replenish the mature monocyte pool for peripheral responses. J. Exp. Med. 213, 22932314

56 Pillay, J. et al. (2010) In vivo labeling with $2 \mathrm{H} 2 \mathrm{O}$ reveals a human neutrophil lifespan of 5.4 days. Blood 116, 625-627

57 Tofts, P.S. et al. (2011) Doubts concerning the recently reported human neutrophil lifespan of 5.4 days. Blood 117, 6050-6052; author reply 6053-6054

58 Li, K.W. et al. (2011) Deuterium and neutrophil kinetics. Blood 117, 6052-6053; author reply 6053-6054

59 Dancey, J.T. et al. (1976) Neutrophil kinetics in man. J. Clin. Invest. 58, 705-715

60 Basu, S. et al. (2002) Evaluation of role of G-CSF in the production, survival, and release of neutrophils from bone marrow into circulation. Blood 100, 854-861

61 Stark, M.A. et al. (2005) Phagocytosis of apoptotic neutrophils regulates granulopoiesis via IL-23 and IL-17. Immunity 22, 285-294

62 Smith, E. et al. (2007) IL-23 is required for neutrophil homeostasis in normal and neutrophilic mice. J. Immunol. 179, 8274-8279

63 Casanova-Acebes, M. et al. (2018) Neutrophils instruct homeostatic and pathological states in naive tissues. J. Exp. Med. 215, 2778-2795

64 Zhang, D. et al. (2015) Neutrophil ageing is regulated by the microbiome. Nature 525, $528-532$

65 He, W. et al. (2018) Circadian Expression of Migratory Factors Establishes LineageSpecific Signatures that Guide the Homing of Leukocyte Subsets to Tissues. Immunity 49, 1175-1190.e7

66 Adrover, J.M. et al. (2019) A Neutrophil Timer Coordinates Immune Defense and Vascular Protection. Immunity 50, 390-402.e10

67 McAlpine, C.S. et al. (2019) Sleep modulates haematopoiesis and protects against atherosclerosis. Nature 566, 383-387

68 Ella, K. et al. (2016) Circadian regulation of human peripheral neutrophils. Brain

Behav. Immun. 57, 209-221

69 Treffers, L.W. et al. (2016) Neutrophils in cancer. Immunol. Rev. 273, 312-328

70 Powell, D.R. and Huttenlocher, A. (2016) Neutrophils in the Tumor 
Microenvironment. Trends Immunol. 37, 41-52

71 Coffelt, S.B. et al. (2016) Neutrophils in cancer: neutral no more. Nat. Rev. Cancer $16,431-446$

72 Silvestre-Roig, C, Fridlender, ZG, Glogauer, M, and Scapini, P. Trends Immunol. (2019) 40, 7: Current issue

73 Gordon, S. and Taylor, P.R. (2005) Monocyte and macrophage heterogeneity. Nat. Rev. Immunol. 5, 953-964

74 Wynn, T.A. et al. (2013) Macrophage biology in development, homeostasis and disease. Nature 496, 445-455

75 Gautier, E.L. and Yvan-Charvet, L. (2014) Understanding macrophage diversity at the ontogenic and transcriptomic levels. Immunol. Rev. 262, 85-95

76 Ginhoux, F. and Guilliams, M. (2016) Tissue-Resident Macrophage Ontogeny and Homeostasis. Immunity 44, 439-449

77 Glass, C.K. and Natoli, G. (2016) Molecular control of activation and priming in macrophages. Nat. Immunol. 17, 26-33

78 Fridlender, Z.G. et al. (2009) Polarization of tumor-associated neutrophil phenotype by TGF-beta: "N1" versus "N2" TAN. Cancer Cell 16, 183-194

79 Jablonska, J. et al. (2010) Neutrophils responsive to endogenous IFN-beta regulate tumor angiogenesis and growth in a mouse tumor model. J. Clin. Invest. 120, 1151-1164

80 Sagiv, J.Y. et al. (2015) Phenotypic diversity and plasticity in circulating neutrophil subpopulations in cancer. Cell Rep 10, 562-573

81 Bauer, S. et al. (2007) Proteinase 3 and CD177 are expressed on the plasma membrane of the same subset of neutrophils. J. Leukoc. Biol. 81, 458-464

82 Amirbeagi, F. et al. (2015) Olfactomedin-4 autoantibodies give unusual c-ANCA staining patterns with reactivity to a subpopulation of neutrophils. J. Leukoc. Biol. 97, 181189

83 Christoffersson, G. et al. (2012) VEGF-A recruits a proangiogenic MMP-9-delivering neutrophil subset that induces angiogenesis in transplanted hypoxic tissue. Blood 120, 46534662

84 Massena, S. et al. (2015) Identification and characterization of VEGF-A-responsive neutrophils expressing CD49d, VEGFR1, and CXCR4 in mice and humans. Blood 126, 2016-2026

85 Matsushima, H. et al. (2013) Neutrophil differentiation into a unique hybrid population exhibiting dual phenotype and functionality of neutrophils and dendritic cells. Blood 121, 1677-1689

86 Singhal, S. et al. (2016) Origin and Role of a Subset of Tumor-Associated Neutrophils with Antigen-Presenting Cell Features in Early-Stage Human Lung Cancer. Cancer Cell 30, $120-135$

87 de Oliveira, S. et al. (2016) Neutrophil migration in infection and wound repair: going forward in reverse. Nat. Rev. Immunol. 16, 378-391

88 Rivera, A. et al. (2016) Innate cell communication kick-starts pathogen-specific immunity. Nat. Immunol. 17, 356-363

89 Silvestre-Roig, C. et al. (2016) Neutrophil heterogeneity: implications for homeostasis and pathogenesis. Blood 127, 2173-2181

90 Hellebrekers, P. et al. (2018) Neutrophil phenotypes in health and disease. Eur. J.

Clin. Invest. 48 Suppl 2, e12943

91 Muller-Sieburg, C.E. et al. (2012) Stem cell heterogeneity: implications for aging and regenerative medicine. Blood 119, 3900-3907

92 Geiger, H. et al. (2013) The ageing haematopoietic stem cell compartment. Nat. Rev. Immunol. 13, 376-389 
93 de Haan, G. and Lazare, S.S. (2018) Aging of hematopoietic stem cells. Blood 131, 479-487

94 Jaiswal, S. et al. (2014) Age-related clonal hematopoiesis associated with adverse outcomes. N. Engl. J. Med. 371, 2488-2498

95 Genovese, G. et al. (2014) Clonal hematopoiesis and blood-cancer risk inferred from blood DNA sequence. N. Engl. J. Med. 371, 2477-2487

96 Xie, M. et al. (2014) Age-related mutations associated with clonal hematopoietic expansion and malignancies. Nat. Med. 20, 1472-1478

97 McKerrell, T. et al. (2015) Leukemia-associated somatic mutations drive distinct patterns of age-related clonal hemopoiesis. Cell Rep 10, 1239-1245

98 Zink, F. et al. (2017) Clonal hematopoiesis, with and without candidate driver mutations, is common in the elderly. Blood 130, 742-752

99 Jaiswal, S. et al. (2017) Clonal Hematopoiesis and Risk of Atherosclerotic

Cardiovascular Disease. N. Engl. J. Med. 377, 111-121

100 Steensma, D.P. et al. (2015) Clonal hematopoiesis of indeterminate potential and its distinction from myelodysplastic syndromes. Blood 126, 9-16

101 Grivennikov, S.I. et al. (2010) Immunity, inflammation, and cancer. Cell 140, 883-

899

102 Pietras, E.M. (2017) Inflammation: a key regulator of hematopoietic stem cell fate in health and disease. Blood 130, 1693-1698

103 Aviv, A. and Levy, D. (2019) Hemothelium, Clonal Hematopoiesis of Indeterminate Potential, and Atherosclerosis. Circulation 139, 7-9

104 Gazit, R. et al. (2008) Hematopoietic stem cells and the aging hematopoietic system. Semin. Hematol. 45, 218-224

105 Bratic, A. and Larsson, N.-G. (2013) The role of mitochondria in aging. J. Clin. Invest. $123,951-957$

106 Soulier, J. (2014) When old hematopoietic stem cells get damaged. Cell Stem Cell 15, 399-400

107 Chandel, N.S. et al. (2016) Metabolic regulation of stem cell function in tissue homeostasis and organismal ageing. Nat. Cell Biol. 18, 823-832

108 Shlush, L.I. (2018) Age-related clonal hematopoiesis. Blood 131, 496-504

109 Busque, L. et al. (2018) Concise Review: Age-Related Clonal Hematopoiesis: Stem

Cells Tempting the Devil. Stem Cells 36, 1287-1294

110 Fuster, J.J. and Walsh, K. (2018) Somatic Mutations and Clonal Hematopoiesis: Unexpected Potential New Drivers of Age-Related Cardiovascular Disease. Circ. Res. 122, 523-532

111 Ebert, B.L. and Libby, P. (2018) Clonal Hematopoiesis Confers Predisposition to Both Cardiovascular Disease and Cancer: A Newly Recognized Link Between Two Major Killers. Ann. Intern. Med. 169, 116-117

112 Jeong, M. et al. (2018) Loss of Dnmt3a Immortalizes Hematopoietic Stem Cells In Vivo. Cell Rep 23, 1-10

113 Challen, G.A. et al. (2011) Dnmt3a is essential for hematopoietic stem cell differentiation. Nat. Genet. 44, 23-31

114 Moran-Crusio, K. et al. (2011) Tet2 loss leads to increased hematopoietic stem cell self-renewal and myeloid transformation. Cancer Cell 20, 11-24

115 Quivoron, C. et al. (2011) TET2 inactivation results in pleiotropic hematopoietic abnormalities in mouse and is a recurrent event during human lymphomagenesis. Cancer Cell 20, 25-38

116 Beerman, I. and Rossi, D.J. (2015) Epigenetic Control of Stem Cell Potential during Homeostasis, Aging, and Disease. Cell Stem Cell 16, 613-625 
117 Stienstra, R. et al. (2017) Specific and Complex Reprogramming of Cellular Metabolism in Myeloid Cells during Innate Immune Responses. Cell Metab. 26, 142-156 118 Oburoglu, L. et al. (2014) Glucose and glutamine metabolism regulate human hematopoietic stem cell lineage specification. Cell Stem Cell 15, 169-184

119 Yvan-Charvet, L. et al. (2010) ATP-binding cassette transporters and HDL suppress hematopoietic stem cell proliferation. Science 328, 1689-1693

120 Nagareddy, P.R. et al. (2013) Hyperglycemia promotes myelopoiesis and impairs the resolution of atherosclerosis. Cell Metab. 17, 695-708

121 Sarrazy, V. et al. (2016) Disruption of Glut1 in Hematopoietic Stem Cells Prevents Myelopoiesis and Enhanced Glucose Flux in Atheromatous Plaques of ApoE(-/-) Mice. Circ. Res. 118, 1062-1077

122 Westerterp, M. et al. (2012) Regulation of hematopoietic stem and progenitor cell mobilization by cholesterol efflux pathways. Cell Stem Cell 11, 195-206

$123 \mathrm{Gu}$, Q. et al. (2019) AIBP-mediated cholesterol efflux instructs hematopoietic stem and progenitor cell fate. Science DOI: 10.1126/science.aav1749

124 Mitroulis, I. et al. (2018) Modulation of Myelopoiesis Progenitors Is an Integral Component of Trained Immunity. Cell 172, 147-161.e12

125 Christ, A. et al. (2018) Western Diet Triggers NLRP3-Dependent Innate Immune Reprogramming. Cell 172, 162-175.e14

126 Nagareddy, P.R. et al. (2014) Adipose tissue macrophages promote myelopoiesis and monocytosis in obesity. Cell Metab. 19, 821-835

127 van den Berg, S.M. et al. (2016) Diet-induced obesity in mice diminishes hematopoietic stem and progenitor cells in the bone marrow. FASEB J. 30, 1779-1788

128 Tall, A.R. and Yvan-Charvet, L. (2015) Cholesterol, inflammation and innate immunity. Nat. Rev. Immunol. 15, 104-116

129 Stiekema, L.C.A. et al. (2017) The maturation of a "neural-hematopoietic" inflammatory axis in cardiovascular disease. Curr. Opin. Lipidol. 28, 507-512

130 Yvan-Charvet, L. and Swirski, F.K. (2018) Is defective cholesterol efflux an integral inflammatory component in myelopoiesis-driven cardiovascular diseases? Eur. Heart J. 39, 2168-2171

131 Deininger, M.W.N. et al. (2017) Turning the tide in myelodysplastic/myeloproliferative neoplasms. Nat. Rev. Cancer 17, 425-440

132 Bowman, R.L. et al. (2018) Clonal Hematopoiesis and Evolution to Hematopoietic Malignancies. Cell Stem Cell 22, 157-170

133 Fossati, G. et al. (2003) The mitochondrial network of human neutrophils: role in chemotaxis, phagocytosis, respiratory burst activation, and commitment to apoptosis. $J$. Immunol. 170, 1964-1972

134 Maianski, N.A. et al. (2004) Functional characterization of mitochondria in neutrophils: a role restricted to apoptosis. Cell Death Differ. 11, 143-153

135 Riffelmacher, T. et al. (2017) Autophagy-Dependent Generation of Free Fatty Acids Is Critical for Normal Neutrophil Differentiation. Immunity 47, 466-480.e5

136 Rožman, S. et al. (2015) The generation of neutrophils in the bone marrow is controlled by autophagy. Cell Death Differ. 22, 445-456

137 Tanimura, A. et al. (2018) Mitochondrial Activity and Unfolded Protein Response are Required for Neutrophil Differentiation. Cell. Physiol. Biochem. 47, 1936-1950

138 Franceschi, C. et al. (2000) Inflamm-aging. An evolutionary perspective on immunosenescence. Ann. N. Y. Acad. Sci. 908, 244-254

139 Gomez, C.R. et al. (2008) Innate immunity and aging. Exp. Gerontol. 43, 718-728

140 Shaw, A.C. et al. (2013) Age-dependent dysregulation of innate immunity. Nat. Rev. Immunol. 13, 875-887 
141 Albright, J.M. et al. (2016) Advanced Age Alters Monocyte and Macrophage Responses. Antioxid. Redox Signal. 25, 805-815

142 Frasca, D. and Blomberg, B.B. (2016) Inflammaging decreases adaptive and innate immune responses in mice and humans. Biogerontology 17, 7-19

143 Franceschi, C. et al. (2018) Inflammaging: a new immune-metabolic viewpoint for age-related diseases. Nat Rev Endocrinol 14, 576-590

144 McHugh, D. and Gil, J. (2018) Senescence and aging: Causes, consequences, and therapeutic avenues. J Cell Biol 217, 65-77

145 Nagata, S. et al. (2010) Autoimmunity and the clearance of dead cells. Cell 140, 619-

630

146 Tabas, I. (2010) Macrophage death and defective inflammation resolution in atherosclerosis. Nat. Rev. Immunol. 10, 36-46

147 Dalli, J. and Serhan, C.N. (2017) Pro-Resolving Mediators in Regulating and Conferring Macrophage Function. Front Immunol 8, 1400

148 Elliott, M.R. and Ravichandran, K.S. (2016) The Dynamics of Apoptotic Cell Clearance. Dev. Cell 38, 147-160

149 Takahashi, R. et al. (2016) Attenuated phagocytosis of secondary necrotic neutrophils by macrophages in aged and SMP30 knockout mice. Geriatr Gerontol Int 16, 135-142

150 Yvan-Charvet, L. et al. (2010) ABCA1 and ABCG1 protect against oxidative stressinduced macrophage apoptosis during efferocytosis. Circ. Res. 106, 1861-1869

151 Park, D. et al. (2011) Continued clearance of apoptotic cells critically depends on the phagocyte Ucp2 protein. Nature 477, 220-224

152 Fond, A.M. et al. (2015) Apoptotic cells trigger a membrane-initiated pathway to increase ABCA1. J. Clin. Invest. 125, 2748-2758

153 Morioka, S. et al. (2018) Efferocytosis induces a novel SLC program to promote glucose uptake and lactate release. Nature 563, 714-718

154 Freemerman, A.J. et al. (2019) Myeloid Slc2a1-Deficient Murine Model Revealed Macrophage Activation and Metabolic Phenotype Are Fueled by GLUT1. J. Immunol. 202, $1265-1286$

155 Zhang, S. et al. (2019) Efferocytosis Fuels Requirements of Fatty Acid Oxidation and the Electron Transport Chain to Polarize Macrophages for Tissue Repair. Cell Metab. 29, 443-456.e5

156 Wang, Y. et al. (2017) Mitochondrial Fission Promotes the Continued Clearance of Apoptotic Cells by Macrophages. Cell 171, 331-345.e22

157 Viaud, M. et al. (2018) Lysosomal Cholesterol Hydrolysis Couples Efferocytosis to Anti-Inflammatory Oxysterol Production. Circ. Res. 122, 1369-1384

158 Han, C.Z. and Ravichandran, K.S. (2011) Metabolic connections during apoptotic cell engulfment. Cell 147, 1442-1445

159 Arnardottir, H.H. et al. (2014) Aging delays resolution of acute inflammation in mice: reprogramming the host response with novel nano-proresolving medicines. J. Immunol. 193, 4235-4244

160 Linehan, E. et al. (2014) Aging impairs peritoneal but not bone marrow-derived macrophage phagocytosis. Aging Cell 13, 699-708

161 Robbins, C.S. and Swirski, F.K. (2010) The multiple roles of monocyte subsets in steady state and inflammation. Cell. Mol. Life Sci. 67, 2685-2693

162 Croker, B.A. et al. (2015) Fight or flight: regulation of emergency hematopoiesis by pyroptosis and necroptosis. Curr. Opin. Hematol. 22, 293-301

163 Boettcher, S. and Manz, M.G. (2017) Regulation of Inflammation- and InfectionDriven Hematopoiesis. Trends Immunol. 38, 345-357

164 Massberg, S. et al. (2007) Immunosurveillance by hematopoietic progenitor cells 
trafficking through blood, lymph, and peripheral tissues. Cell 131, 994-1008

165 Johns, J.L. and Christopher, M.M. (2012) Extramedullary hematopoiesis: a new look at the underlying stem cell niche, theories of development, and occurrence in animals. Vet.

Pathol. 49, 508-523

166 Inra, C.N. et al. (2015) A perisinusoidal niche for extramedullary haematopoiesis in the spleen. Nature 527, 466-471

167 Oda, A. et al. (2018) Niche-induced extramedullary hematopoiesis in the spleen is regulated by the transcription factor Tlx1. Sci Rep 8, 8308

168 Eash, K.J. et al. (2010) CXCR2 and CXCR4 antagonistically regulate neutrophil trafficking from murine bone marrow. J. Clin. Invest. 120, 2423-2431

169 Köhler, A. et al. (2011) G-CSF-mediated thrombopoietin release triggers neutrophil motility and mobilization from bone marrow via induction of Cxcr2 ligands. Blood 117, 4349-4357

170 Semerad, C.L. et al. (2005) G-CSF potently inhibits osteoblast activity and CXCL12 mRNA expression in the bone marrow. Blood 106, 3020-3027

171 Suratt, B.T. et al. (2004) Role of the CXCR4/SDF-1 chemokine axis in circulating neutrophil homeostasis. Blood 104, 565-571

172 Kim, H.K. et al. (2006) G-CSF down-regulation of CXCR4 expression identified as a mechanism for mobilization of myeloid cells. Blood 108, 812-820

173 Devi, S. et al. (2013) Neutrophil mobilization via plerixafor-mediated CXCR4 inhibition arises from lung demargination and blockade of neutrophil homing to the bone marrow. J. Exp. Med. 210, 2321-2336

174 Bajrami, B. et al. (2016) G-CSF maintains controlled neutrophil mobilization during acute inflammation by negatively regulating CXCR2 signaling. J. Exp. Med. 213, 1999-2018 175 Basu, S. et al. (2000) "Emergency" granulopoiesis in G-CSF-deficient mice in response to Candida albicans infection. Blood 95, 3725-3733

176 Kawano, Y. et al. (2017) G-CSF-induced sympathetic tone provokes fever and primes antimobilizing functions of neutrophils via PGE2. Blood 129, 587-597

177 Zhu, Y.P. et al. (2018) Identification of an Early Unipotent Neutrophil Progenitor with Pro-tumoral Activity in Mouse and Human Bone Marrow. Cell Rep 24, 2329-2341.e8

178 Cortez-Retamozo, V. et al. (2012) Origins of tumor-associated macrophages and neutrophils. Proc. Natl. Acad. Sci. U.S.A. 109, 2491-2496

179 Wu, C. et al. (2018) Spleen mediates a distinct hematopoietic progenitor response supporting tumor-promoting myelopoiesis. J. Clin. Invest. 128, 3425-3438

180 Casbon, A.-J. et al. (2015) Invasive breast cancer reprograms early myeloid differentiation in the bone marrow to generate immunosuppressive neutrophils. Proc. Natl. Acad. Sci. U.S.A. 112, E566-575

181 Tu, S. et al. (2008) Overexpression of interleukin-1beta induces gastric inflammation and cancer and mobilizes myeloid-derived suppressor cells in mice. Cancer Cell 14, 408-419 182 Engblom, C. et al. (2017) Osteoblasts remotely supply lung tumors with cancerpromoting SiglecFhigh neutrophils. Science 358,

183 Lee, W. et al. (2019) Neutrophils facilitate ovarian cancer premetastatic niche formation in the omentum. J. Exp. Med. 216, 176-194

184. Veglia, F. et al. (2019) Fatty acid transporter protein 2 reprograms neutrophils in cancer. Nature 569, 73-78.

185 Fiedler, K. and Brunner, C. (2012) The role of transcription factors in the guidance of granulopoiesis. Am J Blood Res 2, 57-65

185. 


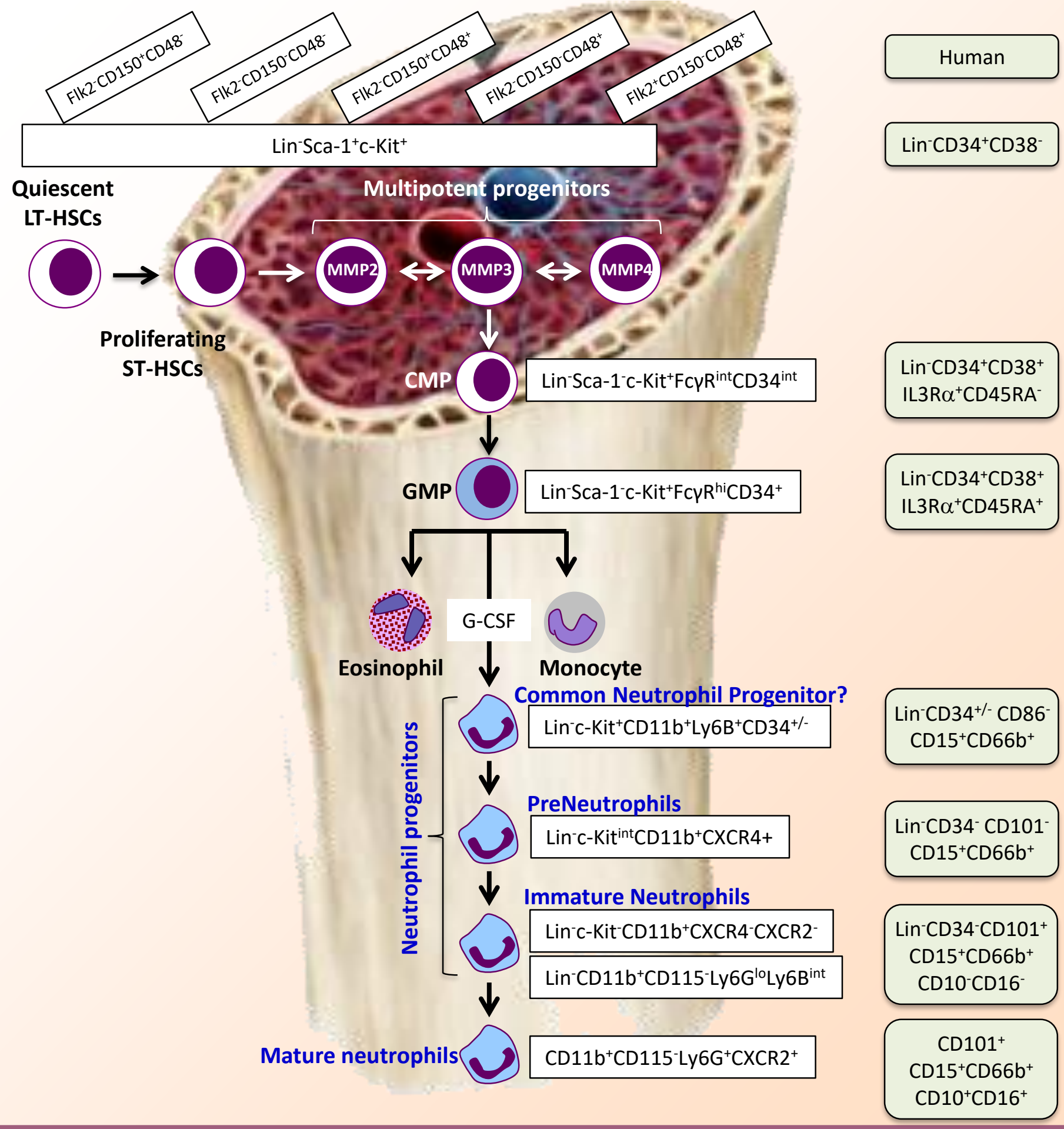

Figure 1 


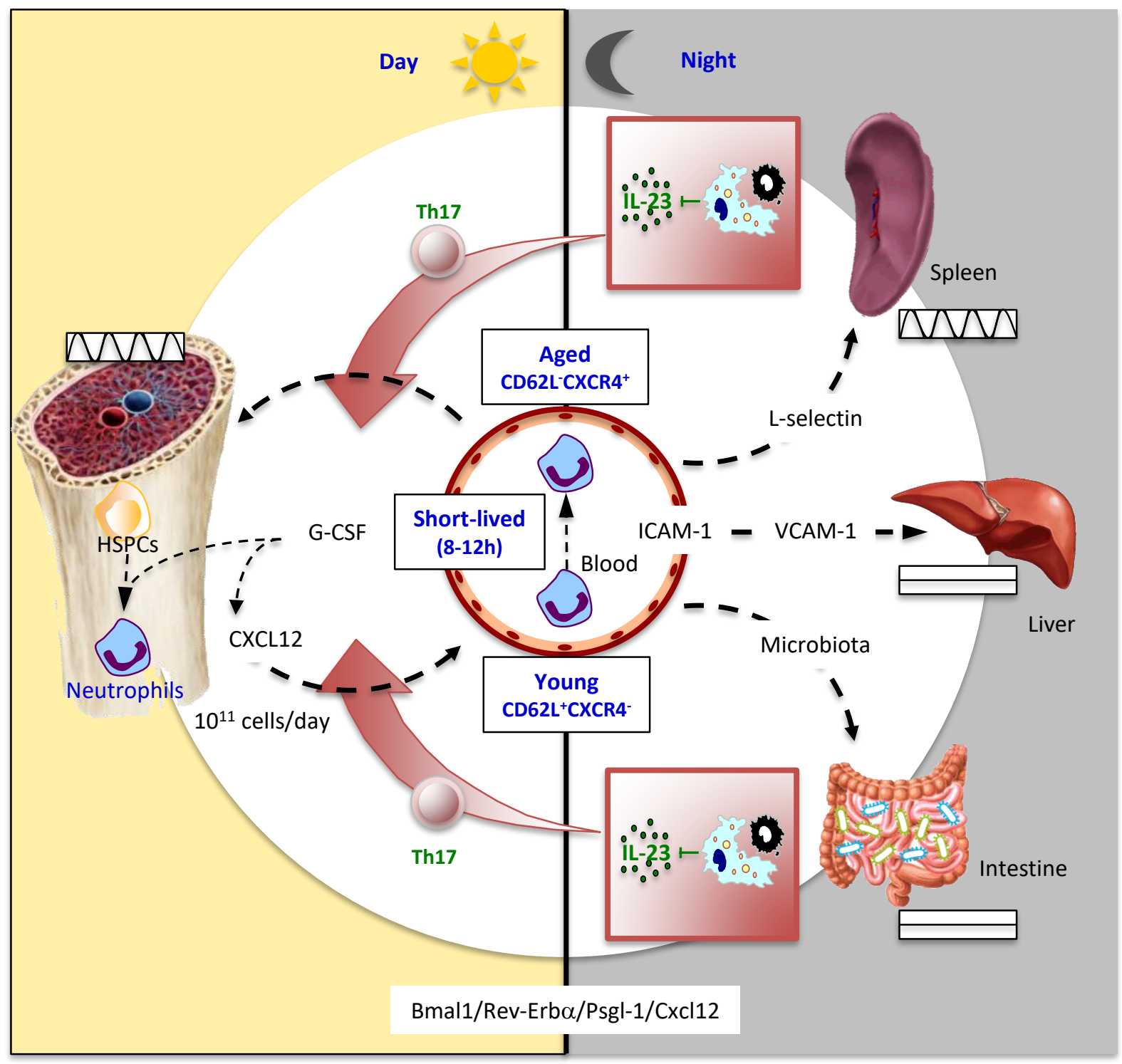

Figure 2 


\section{emergency granulopoiesis}

site of production: bone marrow \& spleen transcription factor: CEBPb

soluble factor: G-CSF, GM-CSF, IL-3, FLT3

\section{tumor-induced granulopoiesis}

site of production: bone marrow \& spleen gene: Retinoblastoma-1 soluble factor: G-CSF, GM-CSF, IL-1b, IL-6

site of production: bone marrow

transcription factor: CEBPa \& CEBPe

soluble factor: G-CSF

homeostatic granulopoiesis 